\title{
Optic Radiation Tractography and Visual Field Deficits in Laser Interstitial Thermal Therapy for Amygdalohippocampectomy in Patients with Mesial Temporal Lobe Epilepsy
}

\author{
Dali Yin $^{\mathrm{a}}$ John A. Thompson ${ }^{\mathrm{a}}$ Cornelia Drees $^{\mathrm{b}}$ Steven G. Ojemann ${ }^{\mathrm{a}}$ \\ Lidia Nagae ${ }^{c}$ Victoria S. Pelak ${ }^{b}$ Aviva Abosch ${ }^{a}$ \\ Departments of ${ }^{a}$ Neurosurgery, ${ }^{b}$ Neurology and ${ }^{c}$ Radiology, University of Colorado, Denver, CO, USA
}

\section{Keywords}

Mesial temporal lobe epilepsy - Laser ablation .

Amygdalohippocampectomy · Visual field defects .

Diffusion tensor tractography - Tractography

\begin{abstract}
Background/Aims: Laser interstitial thermal therapy (LITT) has become an alternative to open-resective surgery for refractory mesial temporal lobe epilepsy (MTLE). Occurrence of visual field defects (VFDs) following open surgery for MTLE is reported at $52-100 \%$. We examined the rate of VFDs following LITT for amygdalohippocampectomy (AHE) and correlated the occurrence of VFDs with damage to the optic radiations, assessed by diffusion tensor tractography (DTI). Methods: We performed a retrospective analysis of 5 patients who underwent LITT-AHE for medically refractory MTLE. We examined the association between VFDs and optic radiation damage by correlating postprocedural visual field testing with qualitative assessment of optic radiation fiber tracts. Results: Postoperative assessments showed that 4 patients had normal visual field testing, and 1 had a right superior quadrantanopsia (20\%). We performed 3-dimensional reconstruction of the optic radiation, laser probe trajectory,
\end{abstract}

and ablation volume. Damage to Meyer's loop was determined consistent with the VFD. Conclusions: Short-term follow-up in our series suggests that laser ablation AHE may be associated with a lower rate of VFD than has been reported for open AHE. Our results suggest that incorporating optic radiation mapping through DTI may preoperatively help to minimize the risk of VFD following laser ablation AHE.

(c) 2017 S. Karger AG, Base

\section{Introduction}

As a minimally invasive surgical technique, magnetic resonance-guided laser interstitial thermal therapy (LITT) for epileptogenic foci has become an alternative treatment option for selected patients with refractory mesial temporal lobe epilepsy (MTLE) [1-4]. Laser ablation amygdalohippocampectomy (AHE) is a relatively new treatment modality for MTLE and has been reported to result in seizure reduction in $77 \%$ of patients with Engle classification, and $54 \%$ of patients were free of disabling seizures [2]. However, patients are less likely to become seizure free with LITT when compared with open resection that resulted in $60-80 \%$ seizure freedom [5-8]. LITT

\section{KARGER}

(C) 2017 S. Karger AG, Basel

E-Mail karger@karger.com

www.karger.com/sfn
Aviva Abosch, MD, $\mathrm{PhD}$

Department of Neurosurgery

University of Colorado, Denver, Campus Box C307

12631 E. 17th Ave, Aurora, CO 80045 (USA)

E-Mail aviva.abosch@ucdenver.edu 
Table 1. MRI parameters used for procedural imaging (clinical Philips Achieva 1.5-T MRI)

\begin{tabular}{|c|c|c|c|c|}
\hline & T1-weighted 3-D & T2-weighted & T2-weighted FLAIR & DWI \\
\hline Repetition time, ms & 7.1 & 3,000 & 11,000 & 3,429 \\
\hline Echo time, ms & 3.2 & 90 & 140 & 71 \\
\hline Inversion time, $\mathrm{ms}$ & 947 & 90 & 2,800 & n.a. \\
\hline Inversion pulse angle, degrees & 8 & n.a. & 90 & n.a. \\
\hline Matrix & $256 \times 256$ & $320 \times 178$ & $256 \times 171$ & $112 \times 87$ \\
\hline Field of view, mm & $256 \times 256$ & $240 \times 180$ & $230 \times 194$ & $230 \times 230$ \\
\hline Bandwidth, $\mathrm{Hz} / \mathrm{Px}$ & 241 & 64.5 & 373.8 & 17.7 \\
\hline Orientation & Axial & Axial & Axial & Axial \\
\hline Slices & $214 \times 1 \mathrm{~mm}$ & $32 \times 2 \mathrm{~mm}$ & $28 \times 1.5 \mathrm{~mm}$ & $28 \times 1.5 \mathrm{~mm}$ \\
\hline Acquisition time, min & 13 & 7 & 4 & 0.2 \\
\hline
\end{tabular}

has certain advantages over open surgery: LITT can be used to precisely target the epileptogenic focus and deeply located seizure foci that would not be amenable to open surgery with minimal disruption of healthy brain tissue $[3,4]$, potentially decreases the risks and complications associated with open surgery $[1,2]$, and reduces hospital stay and costs [2-5].

The visual field defect (VFD) is a common complication following temporal lobe resections for medically intractable MTLE and has been reported to occur in 52$100 \%$ of such patients postoperatively, due to injury to the anterior portion of the optic radiation [9-12]. Functionally significant VFDs were noted in $4-50 \%$ of patients after temporal lobectomy, resulting in their inability to drive and adversely affecting quality of life despite seizure freedom [12-15]. VFDs were also found in $50-62.5 \%$ of patients after radiosurgery for MTLE [16, 17]. Laser ablation AHE also carries risks, but the relationship between VFDs following laser ablation AHE and the possible damage to optic radiation in patients with MTLE is unknown. In this study, we examined the incidence of VFD in a series of patients who underwent laser ablation AHE for intractable MTLE and correlated this deficit with damage to optic radiations, assessed by diffusion tensor imaging (DTI) tractography.

\section{Materials and Methods}

\section{Patient Information}

All procedures, including collection and review of data and analyses, were conducted according to protocols approved by the Colorado Multi-Institutional Review Board. Data collection and analysis were based on review of medical records.

We retrospectively reviewed the clinical data of 5 consecutive patients who underwent laser ablation AHE for medically refractory MTLE between June and December 2015. There were 3 men and 2 women, with a mean age of 45 years (range, 28-69). The standard preoperative evaluation for the procedure included electroencephalography (EEG), video EEG, magnetic resonance imaging (MRI), functional MRI, DTI, positron emission tomography, intracarotid amobarbital (Wada) test, neuropsychological evaluation, and intracranial EEG recordings prior to surgery, if deemed clinically necessary. Functional MRI and Wada testing were performed to lateralize language dominance and predict the risk of postoperative memory deficits. Each case was discussed at the University of Colorado multidisciplinary Epilepsy Patient Care Conference, which provided consensus recommendations regarding surgery. Intracranial electrode monitoring was performed in $1 \mathrm{pa}-$ tient in this study. All 5 patients underwent laser ablation AHE.

The clinical follow-up included assessment of VFDs and seizure outcome by medical records and patient interview. Patients were evaluated 2 weeks after the procedure, and again 2-9 months thereafter. During clinic visits, we collected seizure information, verified medications, and evaluated for complications and quality of life. All patients underwent formal visual field testing using an automated perimetry method 2-8 months after laser ablation AHE.

\section{Conventional MRI and DTI}

Procedural MRI was performed on a clinical Philips Achieva 1.5-T MRI scanner using a Transmit/Receive head coil for the Leksell frame and a Sense Flexible Large head coil for the CRW frame. Specific parameters utilized for T1-weighted, T2-weighted, diffusion-weighted imaging (DWI) and fluid-attenuated inversion recovery (FLAIR) sequences are shown in Table 1. For DTI, a singleshot spin echo diffusion-weighted echo planar imaging sequence was performed on a GE 3.0-T scanner with the following parameters: 32 gradient directions, echo time $84.9 \mathrm{~ms}$, repetition time $16,000 \mathrm{~ms}$, matrix size $128 \times 128$, FOV $240 \mathrm{~mm}$, slice thickness 2.6 $\mathrm{mm}$, bandwidth $250 \mathrm{~Hz} / \mathrm{Px}$, echo train length 1 , Nex of 1, b-maximum of $1,000 \mathrm{~s} / \mathrm{mm}^{2}, 1 \mathrm{~b} 0,53$ slices, no intersection gap, scan time $9 \min 4 \mathrm{~s}$.

\section{Optic Radiation Fiber Tracking}

For reconstruction and visualization of the optic radiation, BrainLab iPlan 3.0 (BrainLab, Feldkirchen, Germany) navigation planning software was used. Tract seeding was placed as a rectangular region of interest (ROI) in the lateral geniculate nucleus 
(LGN) and optic tract ipsilateral to the ablated one, in order to generate optic radiation $[18,19]$. Fiber tract seed ROIs were placed along the optic tract and the LGN ipsilateral to the ablated hemisphere. The LGN was localized posterolateral to the peduncles [18, 19]. A multi-ROI algorithm that included ROIs along the optic tract and the occipital visual cortex (calcarine cortex) was utilized. The 3-dimensional (3-D) optic radiation was generated in the 3-D space of the T1-weighted sequence. For better understanding of the presumed Meyer's loop injury, evaluation of the coregistered images of the 3-D reconstruction of the optic radiation fiber tracts, the laser probe trajectory (obtained from the preprocedural T1weighted images), and the ablation volume of the amygdala hippocampal complex (obtained from the postprocedural T1-weighted contrast images) was performed.

\section{MR-Guided Laser Ablation AHE}

After obtaining informed consent, patients underwent placement of an MR-compatible stereotactic head frame (CRW, Integra, Plainsboro, NJ, USA, or Leksell, Elekta, Stockholm, Sweden) using a local anesthetic. A fiducial localizer was secured to the frame base ring, and a brain CT was obtained for stereotactic localization. Brain MRI was obtained on a clinical Philips Achieva 1.5-T scanner, using a Transmit/Receive head coil for the Leksell frame and a Sense Flexible Large head coil for the CRW frame, including the following sequences: T1-weighted 3-D volumetric postcontrast $\left(1 \mathrm{~mm}^{3}\right)$, T2-weighted postcontrast and T2-weighted FLAIR. Neuronavigational software (BrainLab iPlan, Feldkirchen, Germany) was then used to fuse preoperative stereotactic CT and MR images for trajectory and target planning aimed at the amygdala and hippocampus, on axial, coronal, and sagittal planes. The trajectory planning optimized the parieto-occipital entry point and maximized the length of the laser fiber trajectory through the long axis of the hippocampus and amygdala, while avoiding sulcal entry, detectable vasculature, and choroid plexus.

Patients were then transported to the operating room, and the laser catheter (Visualase; Medtronic Inc., Minneapolis, MN, USA) was inserted under general anesthesia, through a bone anchor placed using a standard stereotactic technique. The distance to target from the top of the anchor bolt was calculated. The laser applicator assembly with polycarbonate cooling catheter and flexible laser optical fiber was prepared. After the anchor cap had been loosened, the catheter - with stylet fully inserted - was lowered through the anchor bolt into the underlying brain. Lateral C-arm visualization was used to verify the laser catheter tip position at the intended target. The inner stylet was then removed, and the laser optical fiber with diffuser tip was inserted to the end of the cooling catheter. The laser applicator assembly was secured in place via the anchor cap on the proximal end of the anchor bolt. The stereotactic arc was then removed, and patients were transported back to the MRI (Philips Achieva 1.5 T) suite with care taken not to damage or dislodge the catheter and laser fiber. Localizing and planning scans were obtained. The targets in the amygdala, and hippocampal head and body were identified. The cooling system and laser were connected to the workstation. Safety regions were designated with temperature limits to minimize off-target thermal damage to surrounding normal tissue. A test dose with $30 \%$ laser power was undertaken to validate MR images, and heating appeared at both the correct target and the tip of the cooling catheter. Thermal ablation AHE was then carried out at 70\% laser power. The duration of laser treatment was based on real-time MR ther-

Visual Field Deficits following Laser

Ablation Amygdalohippocampectomy mography images of the extent of ablation. The laser probe was withdrawn within the cooling catheter as needed, in order to cover a greater extent of the intended target. All patients underwent insertion of a single laser fiber, and were treated with 2-4 contiguous ablation zones along a single trajectory, to achieve a total ablation area involving the amygdala and hippocampus. Ongoing thermal imaging maps and ablation zone were viewed in real time on the laser workstation. The laser tests were performed at $4.50 \mathrm{~W}$ for $14-27 \mathrm{~s}$, and ablations were achieved at $9.00 \mathrm{~W}$ for $63-175 \mathrm{~s}$. Postprocedural MRI sequences including DWI, T2-weighted FLAIR, and postgadolinium contrast T1-weighted sequences were acquired, to check for signal abnormality and postcontrast enhancement outlining the intended lesion volume. In all cases, postgadolinium T1-weighted MR images at the completion of laser ablation disclosed enhancement surrounding the inferior amygdala and hippocampus, posteriorly to the level of the tectal plate. No surrounding abnormalities were noted on imaging. The laser probe and catheter, and the anchor bolt were completely removed, the skin incision was closed and the stereotactic frame removed. $\mathrm{Pa}$ tients were admitted overnight to the neurological intensive care unit for observation, and then discharged with a 1-week oral dexamethasone taper.

\section{Results}

\section{Preoperative Data}

The preoperative evaluation included EEG, video EEG, MRI, Wada test and neuropsychological testing in 5 patients, positron emission tomography in 4 patients, functional MRI and DTI in 2 patients. Typical MRI findings of mesial temporal sclerosis were found in 4 patients. Preoperative seizure frequency ranged from 2 seizures/ month (4 patients) to 5-6 seizures/month. One patient underwent intracranial monitoring with bilateral subtemporal and lateral temporal electrodes, which captured complex partial seizures with right medial temporal onset.

\section{Procedure and Imaging}

Laser ablation AHE was performed on the right side in 3 patients and on the left in 2 . The final ablation zone area was confirmed by immediate postprocedure T2-weighted FLAIR, DWI and contrast-enhanced T1-weighted MR images, as shown in Figure 1. No encroachment of the ablation zone into other structures was seen. No intra- or postoperative hematomas were noted during or after laser ablation procedures.

\section{Clinical Outcome}

Four patients were discharged on postoperative day 1, and 1 patient was admitted for 9 days for intracranial electrode monitoring of seizures, followed by laser ablation 
Fig. 1. Radiographic effects of laser ablation of right AHE. Coronal (a) and sagittal (b) T1-weighted, postcontrast MR images showing the laser ablation area demarcated by linear gadolinium enhancement indicated by arrow(s). Dark line on sagittal image represents laser catheter in place, prior to removal.
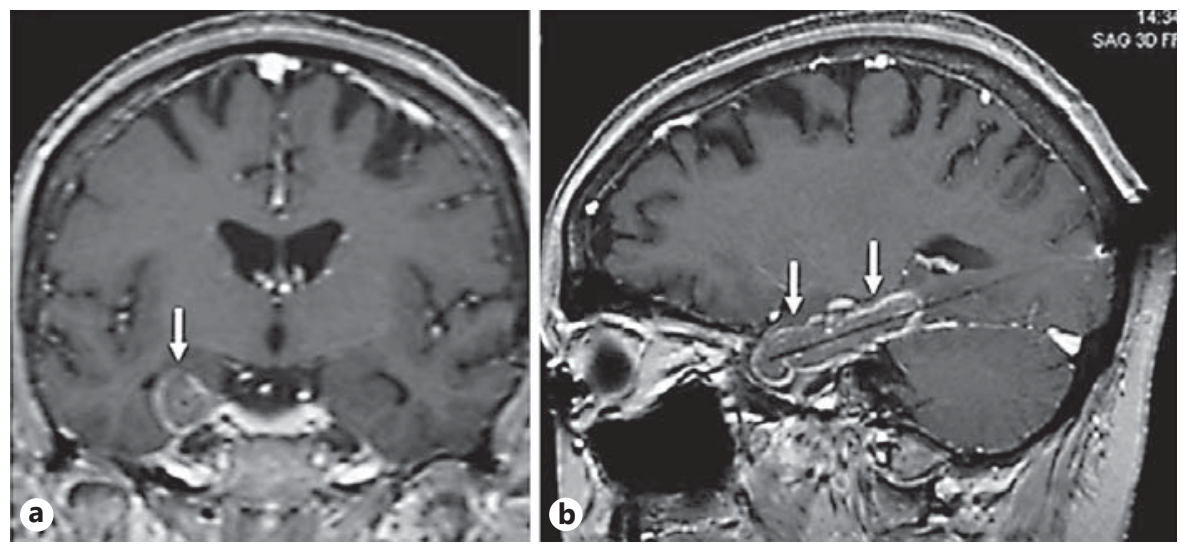

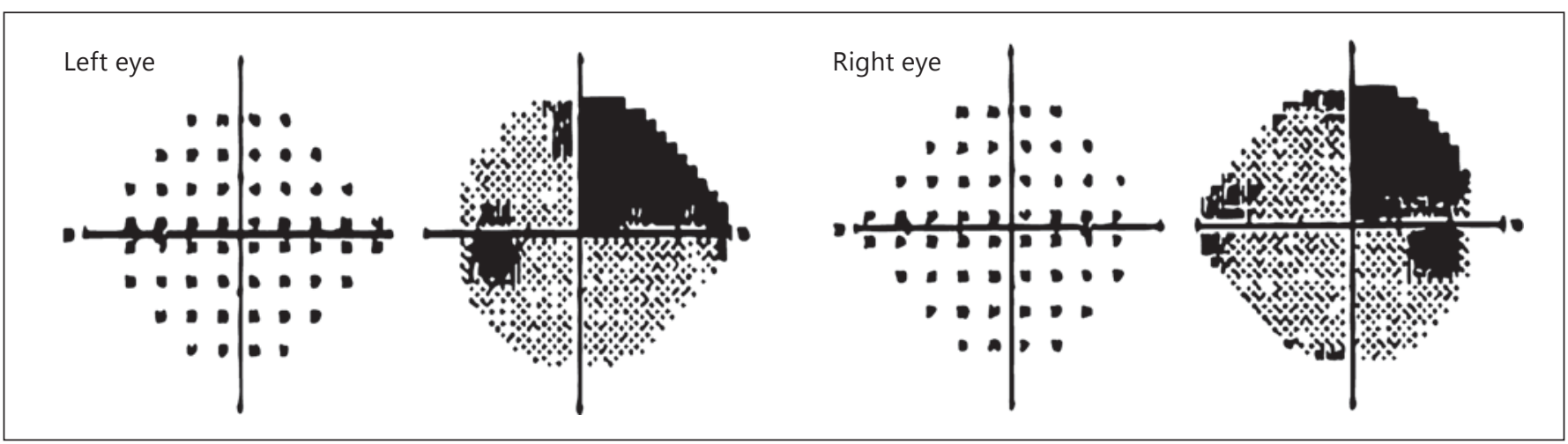

Fig. 2. Visual field test showing postablation right superior quadrantanopsia in 1 patient.

of the seizure onset zone. With follow-up ranging from 3 to 9 (median, 7) months after laser ablation AHE, all patients (100\%) achieved a significant seizure reduction, and 1 patient was seizure free at the last follow-up. EEG measures before and after surgery were not qualitatively different. Postoperatively, no patient reported subjective visual changes or had detectable VFDs based on bedside confrontation testing. All patients underwent formal visual field testing 3-8 months postoperatively. Four patients had normal visual field testing, and 1 demonstrated a right superior quadrantanopsia (20\%) (Fig. 2). No other complications or neuropsychological side effects related to laser ablation were noted. There was no mortality in our study.

\section{3-D Reconstruction of Optic Radiation, Laser Probe \\ Trajectory and Ablation Volume}

With our methods, the white matter fibers of the optic radiation were successfully identified in the patient with right superior quadrantanopsia. The coregistration of the
3-D reconstruction of optic radiation, laser probe trajectory, and ablation volume showed that Meyer's loop was penetrated by the trajectory of the laser catheter (Fig. 3, 4), consistent with the development of a VFD in this patient.

\section{Discussion}

In this study, we performed laser ablation AHE on 5 patients with MTLE and found that the incidence of VFD following laser ablation AHE was much lower (20\%) than the rate reported for open temporal lobe resection (52$100 \%)$ [9-12] and for radiosurgery (50-62.5\%) $[16,17]$. On formal visual field testing, 4 patients had normal visual fields, and 1 patient had right superior quadrantanopsia. Moreover, our data showed that laser ablation AHE significantly reduced the frequency and severity of epilepsy of our patients and was suggestive of a low risk of complications. 
Fig. 3. a DTI tractography demonstrating optic radiations. b, c 3-D reconstruction of optic radiation, laser probe trajectory, and ablation volume showed that Meyer's loop was penetrated by the laser probe (red; colors in the online version only) and involved in the ablation area (orange-yellow) as shown by arrow.
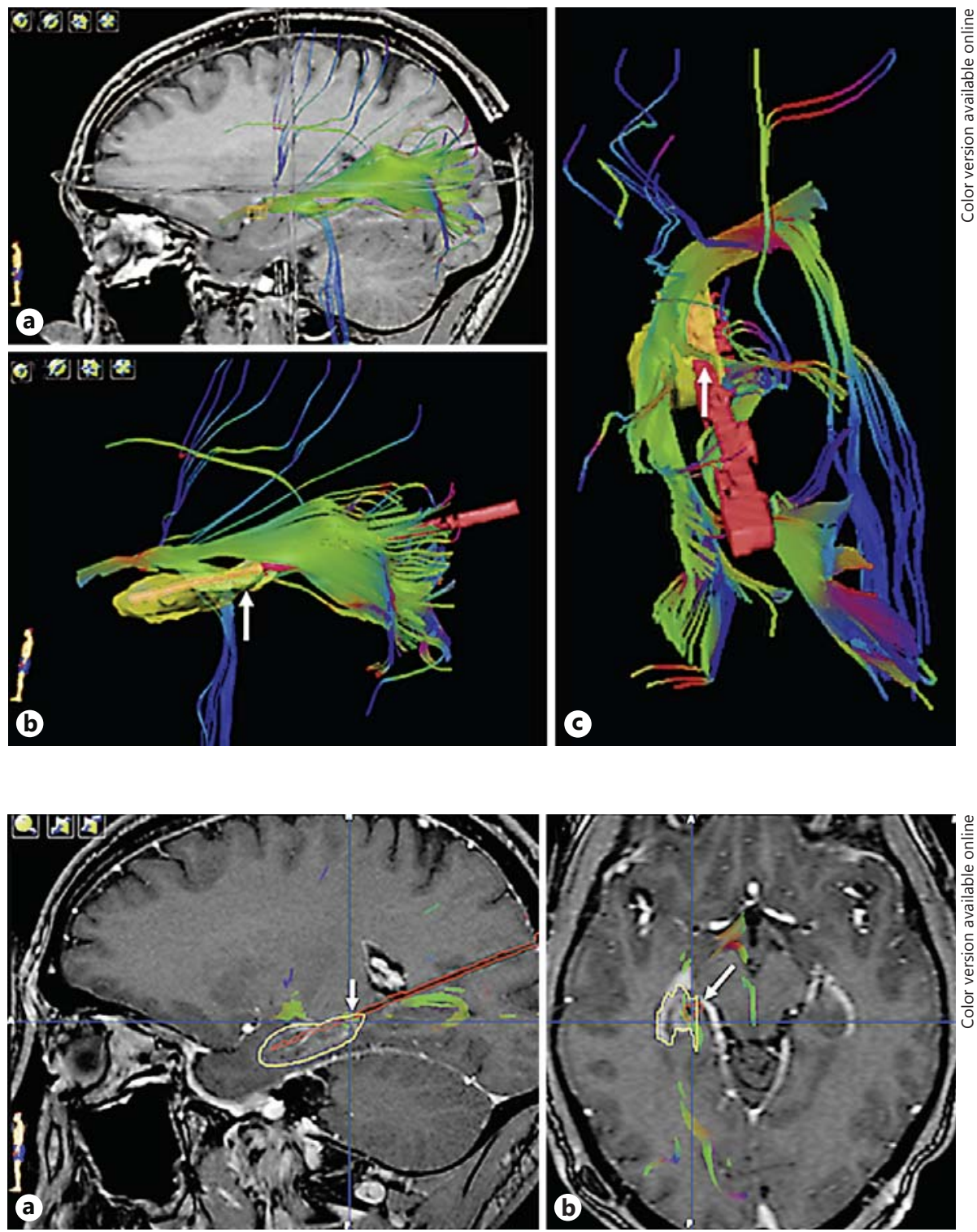

Fig. 4. Sagittal (a) and axial (b) T1-weighted, contrast MR images showing the relation among optic radiation, laser probe trajectory, and laser ablation area. Green (colors in the online version only), optic radiation; red, laser probe trajectory; yellow, laser ablation area.
Drug-resistant epilepsy occurs in approximately one third of epilepsy patients [20-23]. Anterior temporal lobectomy is a well-established treatment for patients with drug-resistant MTLE [24-27] and results in 60-80\% seizure freedom in selected patients $[5,28,29]$, but is associated with adverse effects, such as cognitive impairments and focal neurological deficits [30-36]. Therefore, the balance of effectiveness and possible complications of open resection needs to be taken into account for patients with MTLE. As a minimally invasive surgical technique, laser ablation AHE is an appealing alternative to patients with temporal lobectomy, and offers an effective treatment option for patients with MTLE who are otherwise not eligible for open surgery $[1,2]$. Laser ablation AHE may also play an important role in surgically complex patients who would otherwise require reoperation via open surgery [37], and for whom the risk of complications of re-do open surgery is too high. Previous reports support comparable seizure control rates for laser ablation AHE and open surgery, while the former affords the possibility of lower complication rates $[1,2]$. Laser ablation AHE was found to be safe and effective for MTLE with most patients achieving freedom of disabling seizures, especially patients with mesial temporal sclerosis $[1-4,37,38]$. For example, it has been reported by Willie et al. [2] that " $77 \%$ of patients achieved meaningful seizure reduction, 
of which $54 \%$ were free of disabling seizures." Moreover, improved outcomes have been observed on neuropsychological testing at 6 months and 1 year as compared to open resections $[2,38]$. Laser ablation AHE is performed with simultaneous MRI guidance, which enables realtime monitoring of and feedback from tissue ablation, and has several advantages as compared to temporal lobectomy. It is less invasive, and affords precise targeting of the seizure onset zone with minimal disruption of surrounding healthy brain tissue, and reduces hospital stay and costs due to low complication rates and minimal recovery time $[3,4]$. Laser ablation AHE may also decrease the concerns regarding risk and fears about open brain surgery of patients and referring physicians [3].

It is important to note, however, that laser ablation AHE is not without risk. Concerns persist regarding its surgical complications, including VFD, which is due to the course of the optic radiations within the temporal and occipital lobe $[18,19]$. Waseem et al. [1] reported that $28.6 \%$ ( 2 of 7 ) patients with laser ablation AHE had partial VFD. Willie et al. [2] found VFD in 1 of 13 patients with laser ablation AHE, which resulted from deviated insertion of a stereotactic aligning rod. Our experience suggests that laser ablation AHE is associated with a lower rate of VFD, seen in only 1 of 5 patients as compared to open surgery - a finding that is consistent with previously published reports $[1,2]$. The postoperative visual field loss was in the upper visual field in our patient, suggesting damage to the optic radiations within Meyer's loop. Tractography confirmed this assertion, demonstrating that the laser catheter penetrated Meyer's loop (Fig. 3, 4).

The optic radiation is a massive fiber bundle emanating from the LGN and projecting to the primary visual cortex [39-41]. Fibers from the inferior visual field run directly posteriorly from the LGN, whereas the fibers from the superior field first pass anteriorly and laterally over the temporal horn of the lateral ventricle in Meyer's loop, before turning posteriorly into the occipital lobe [42]. This anatomical relationship between the optic radiations contained in Meyer's loop and the temporal horn of the lateral ventricle place the superior quadrant visual field fibers at greater risk of injury during laser ablation AHE. However, VFD after laser ablation AHE may be related to damage of any part of the optic radiation located in the temporal and occipital lobes by a laser ablation procedure. anatomical MRI sequences. However, the advances in DTI-based tractography provide us with useful information about optic radiations $[18,19]$. This technique allows alignment of preoperative optic radiation tracts onto pre-

and postoperative MR images, with the potential to reduce damage to optic radiations by allowing for modification of laser trajectory, thereby reducing the risk of developing VFDs. Postoperatively, in case of VFDs, a correlation with damage to the optic radiation is also possible. Given the variation in location and course of Meyer's loop and the optic radiations between individuals [19, 41], DTI is extremely useful for preoperative laser probe trajectory planning. Tractography has been used to successfully predict visual field outcome in patients with temporal lobectomy [42, 43-46]. So far there are no reports studying the relationships among VFDs, optic radiation, laser probe trajectory and ablation volume for laser ablation AHE. Therefore, we recommend that DTI tractography be performed to delineate the optic radiation for surgical planning before laser ablation AHE, and predict possible visual consequences of the procedure.

Limitations of this study include its retrospective nature, the small patient number and short follow-up. Although these results suggest that there is a lower risk of VFD associated with LITT-AHE for patients with MTLE as opposed to open resection, further study is needed to establish that this risk reduction will generalize to a larger study sample.

\section{Conclusions}

Our findings support previous reports that laser ablation AHE is associated with a lower rate of VFD than anterior temporal resection. Preoperative visualization of the optic radiation, including Meyer's loop, by DTI tractography may help reduce the risk of VFD following laser ablation AHE. It would be advantageous to incorporate the optic radiation of DTI tractography into MR images to guide surgical planning and identify patients at risk for postsurgical VFDs. A larger series with long-term followup is necessary to assess the robustness of this finding.

The optic radiation cannot be identified on existing

References
Stereotact Funct Neurosurg 2017;95:107-113 DOI: $10.1159 / 000454866$
Yin/Thompson/Drees/Ojemann/Nagae/ Pelak/Abosch
1 Waseem H, Osborn KE, Schoenberg MR, Kelley V, Bozorg A, Cabello D, Benbadis SR, Vale FL: Laser ablation therapy: an alternative treatment for medically resistant mesial temporal lobe epilepsy after age 50. Epilepsy Behav 2015;51:152-157.

2 Willie JT, Laxpati NG, Drane DL, Gowda A, Appin C, Hao C, Brat DJ, Helmers SL, Saindane A, Nour SG, Gross RE: Real-time magnetic resonance-guided stereotactic laser amygdalohippocampotomy for mesial temporal lobe epilepsy. Neurosurgery 2014;74:569-585. 
3 Hawasli AH, Bandt SK, Hogan RE, Werner N, Leuthardt EC: Laser ablation as treatment strategy for medically refractory dominant insular epilepsy: therapeutic and functional considerations. Stereotact Funct Neurosurg 2014;92:397-404.

4 Hawasli AH, Bagade S, Shimony JS, MillerThomas M, Leuthardt EC: Magnetic resonance imaging-guided focused laser interstitial thermal therapy for intracranial lesions: single-institution series. Neurosurgery 2013; 73:1007-1017.

5 Chang EF, Englot DJ, Vadera S: Minimally invasive surgical approaches for temporal lobe epilepsy. Epilepsy Behav 2015;47:24-33.

6 Jeha LE, Najm IM, Bingaman WE, Khandwala F, Widdess-Walsh P, Morris HH, et al: Predictors of outcome after temporal lobectomy for the treatment of intractable epilepsy. Neurology 2006;66:1938-1940.

7 Vadera S, Kshettry VR, Klaas P, Bingaman W: Seizure-free and neuropsychological outcomes after temporal lobectomy with amygdalohippocampectomy in pediatric patients with hippocampal sclerosis. J Neurosurg Pediatr 2012;10:103-107.

8 Englot DJ, Chang EF: Rates and predictors of seizure freedom in resective epilepsy surgery: an update. Neurosurg Rev 2014;37:389-404; discussion 404-405.

9 Tecoma ES, Laxer KD, Barbaro NM, Plant GT: Frequency and characteristics of visual field deficits after surgery for mesial temporal sclerosis. Neurology 1993;43:1235-1238.

10 Hughes TS, Abou-Khalil B, Lavin PJ, Fakhoury T, Blumenkopf B, Donahue SP: Visual field defects after temporal lobe resection: a prospective quantitative analysis. Neurology 1999;53:167-172.

11 Egan RA, Shults WT, So N, Burchiel K, Kellogg JX, Salinsky M: Visual field deficits in conventional anterior temporal lobectomy versus amygdalohippocampectomy. Neurology 2000;55:1818-1822.

12 Bjellvi J, Rydenhag B, Malmgren K: Visual field defects and permission to drive in adults after temporal lobe resections for epilepsy. Epilepsia 2014;55:4-246.

13 Manji H, Plant GT: Epilepsy surgery, visual fields, and driving: a study of the visual field criteria for driving in patients after temporal lobe epilepsy surgery with a comparison of Goldmann and Esterman perimetry. J Neurol Neurosurg Psychiatry 2000;68:80-82.

14 Pathak-Ray V, Ray A, Walters R, Hatfield R: Detection of visual field defects in patients after anterior temporal lobectomy for mesial temporal sclerosis - establishing eligibility to drive. Eye (Lond) 2002;16:744-748.

15 Jeelani NU, Jindahra P, Tamber MS, Poon TL, Kabasele P, James-Galton M, Stevens J, Duncan J, McEvoy AW, Harkness W, Plant GT: Hemispherical asymmetry in the Meyer's Loop: a prospective study of visual-field deficits in 105 cases undergoing anterior temporal lobe resection for epilepsy. J Neurol Neurosurg Psychiatry 2010;81:985-991.
16 Hensley-Judge $\mathrm{H}$, Quigg M, Barbaro NM, Newman SA, Ward MM, Chang EF, Broshek DK, Lamborn KR, Laxter KD, Garcia P, Heck CN, Kondziolka D, Beach R, Salanova V, Goodman R: Visual field defects after radiosurgery for mesial temporal lobe epilepsy. Epilepsia 2013;54:1376-1380.

17 Regis J, Rey M, Bartolomei F, Vladyka V, Liscak R, Schrottner O, Pendl G: Gamma knife surgery in mesial temporal lobe epilepsy: a prospective multicenter study. Epilepsia 2004;45:504-515.

18 Lilja Y, Nilsson DT: Strengths and limitations of tractography methods to identify the optic radiation for epilepsy surgery. Quant Imaging Med Surg 2015;5:288-299.

19 Sherbondy AJ, Dougherty RF, Napel S, Wandell BA: Identifying the human optic radiation using diffusion imaging and fiber tractography. J Vis 2009;8:12.1-12.11.

20 Kwan P, Brodie MJ: Early identification of refractory epilepsy. N Engl J Med 2000;342: 314-319.

21 Devinsky O, Pacia S: Epilepsy surgery. Neurol Clin 1993;11:951-971.

22 Engel J Jr: Surgery for seizures. N Engl J Med 1996;334:647-652.

23 Connor DE Jr, Nixon M, Nanda A, Guthikonda B: Vagal nerve stimulation for the treatment of medically refractory epilepsy: a review of the current literature. Neurosurg Focus 2012;32:E12.

24 Kwan P, Schachter S, Brodie M: Drug-resistant epilepsy. N Engl J Med 2011;365:919-926.

25 Duncan JS, Sander JW, Sisodiya SM, Walker MC: Adult epilepsy. Lancet 2006;367:10871100.

26 Marson A, Jacoby A, Johnson A, et al: Immediate versus deferred antiepileptic drug treatment for early epilepsy and single seizures: a randomised controlled trial. Lancet 2005;365: 2007-2013.

27 Hauser WA, Rich SS, Lee JR, Annegers JF, Anderson VE: Risk of recurrent seizures after two unprovoked seizures. N Engl J Med 1998; 338:429-434.

28 Wiebe S, Blume WT, Girvin JP, Eliasziw M: Effectiveness and efficiency of Surgery for Temporal Lobe Epilepsy Study Group. A randomized, controlled trial of surgery for temporal-lobe epilepsy. N Engl J Med 2001;345: 311-318.

29 Duncan JS: Epilepsy surgery. Clin Med 2007; 7:137-142.

30 Jette N, Reid AY, Wiebe S: Surgical management of epilepsy. CMAJ 2014;186:997-1003.

31 Hader WJ, Tellez-Zenteno J, Metcalfe A, et al: Complications of epilepsy surgery - a systematic review of focal surgical resections and invasive EEG monitoring. Epilepsia 2013;54: 840-847.

32 Spencer S, Huh L: Outcomes of epilepsy surgery in adults and children. Lancet Neurol 2008:7:525-537.

33 Lutz MT, Clusmann H, Elger CE, Schramm J, Helmstaedter C: Neuropsychological outcome after selective amygdalohippocampec- tomy with transsylvian versus transcortical approach: a randomized prospective clinical trial of surgery for temporal lobe epilepsy. Epilepsia 2004;45:809-816.

34 Crane J, Milner B: Do I know you? Face perception and memory in patients with selective amygdalo-hippocampectomy. Neuropsychologia 2002;40:530-538.

35 Drane DL, et al: Category-specific naming and recognition deficits in temporal lobe epilepsy surgical patients. Neuropsychologia 2008;46:1242-1255.

36 Seidenberg M, et al: Neuropsychological outcome following anterior temporal lobectomy in patients with and without the syndrome of mesial temporal lobe epilepsy. Neuropsychology 1998;12:303-316.

37 Lewis EC, Weil AG, Duchowny M, Bhatia S, Ragheb J, Miller I: MR-guided laser interstitial thermal therapy for pediatric drug-resistant lesional epilepsy. Epilepsia 2015;35:1-9.

38 Drane DL, et al: Temporal lobe epilepsy surgical patients undergoing MRI-guided stereotactic laser ablation exhibit better episodic memory outcome as compared to standard surgical approaches. Epilepsia 2013;54:20.

39 Cushing $\mathrm{H}$ : The field defects produced by temporal lobe lesions. Brain 1922;44:341396.

40 Meyer A: The connections of the occipital lobes and the present status of the cerebral visual affections. Trans Assoc Am Physicians 1907;22:7-16.

41 Ebeling U, Reulen HJ: Neurosurgical topography of the optic radiation in the temporal lobe. Acta Neurochir 1988;92:29-36.

42 Winston GP, Daga P, Stretton J, Modat M, Symms MR, McEvoy AW, Ourselin S, Duncan JS: Optic radiation tractography and vision in anterior temporal lobe resection. Ann Neurol 2012;71:334-341.

43 Taoka T, Sakamoto M, Nakagawa H, Nakase $\mathrm{H}$, Iwasaki S, Takayama K, Taoka K, Hoshida T, Sakaki T, Kichikawa K: Diffusion tensor tractography of the Meyer loop in cases of temporal lobe resection for temporal lobe epilepsy: correlation between postsurgical visual field defect and anterior limit of Meyer loop on tractography. AJNR Am J Neuroradiol 2008;29:1329-1334.

44 Chen X, Weigel D, Ganslandt O, Buchfelder M, Nimsky C: Prediction of visual field deficits by diffusion tensor imaging in temporal lobe epilepsy surgery. Neuroimage 2009;45: 286-297.

45 Yogarajah M, Focke NK, Bonelli S, Cercignani M, Acheson J, Parker GJ, Alexander DC, McEvoy AW, Symms MR, Koepp MJ, Duncan JS: Defining Meyer's loop-temporal lobe resections, visual field deficits and diffusion tensor tractography. Brain 2009;132:1656-1668.

46 Powell HW, Parker GJ, Alexander DC, Symms MR, Boulby PA, Wheeler-Kingshott CA, Barker GJ, Koepp MJ, Duncan JS: MR tractography predicts visual field defects following temporal lobe resection. Neurology 2005;65:596-599.
Visual Field Deficits following Laser Ablation Amygdalohippocampectomy
Stereotact Funct Neurosurg 2017;95:107-113 